Cahiers de recherches médiévales

La Noblesse en question (XIII $-\mathrm{XV}^{\mathrm{e}} \mathrm{s}$.)

\title{
Membra diaboli
}

Remarques sur le statut et l'imaginaire du corps sorcier au $\mathrm{XV}^{\mathrm{e}}$ siècle

\section{Franck Mercier}

\section{(2) OpenEdition}

Journals

Édition électronique

URL : https://journals.openedition.org/crm/766

DOI : $10.4000 / \mathrm{crm} .766$

ISSN : 1955-2424

Éditeur

Honoré Champion

Édition imprimée

Date de publication : 30 décembre 2006

Pagination : 181-193

ISSN : 1272-9752

Référence électronique

Franck Mercier, «Membra diaboli », Cahiers de recherches médiévales [En ligne], 13 | 2006, mis en ligne le 27 novembre 2009, consulté le 15 décembre 2022. URL : http://journals.openedition.org/crm/766 ; DOI : https://doi.org/10.4000/crm.766 


\section{酮RM}

\section{Membra diaboli
Remarques sur le statut et l'imaginaire du corps sorcier au $X V^{\mathrm{e}}$ siècle}

L'An 1460, le $26^{\text {e }}$ jour d'aoust, en la ville de Mantes fust ards et son corps ramené en pouldre et la pouldre par jugement jettée au vent, ung nommé Noël Ferre, natif d'Amiens, comme il disoit, de l'âge de 28 ans, lequel confessa d'estre sorcier et (...) d'avoir esté par quatre fois en vaulderie, en laquelle vaulderie il avoit adoré le diable en forme de boucq ${ }^{1}$.

Pour la justice en charge de la persécution de la sorcellerie, la cause semble entendue vers le milieu du $\mathrm{XV}^{\mathrm{e}}$ siècle : le corps sorcier est d'abord un corps à brûler, une chair à bûcher. Comme l'hérétique relaps ou impénitent, l'individu reconnu coupable de s'être rendu au sabbat (c'est le sens du mot «vaulderie») doit périr par le feu de façon que son corps soit «ramené en pouldre» et ses cendres dispersées au vent. Il s'agit aussi d'une épreuve de vérité : à l'opposé du corps du martyr dont le supplice laisse toujours subsister un reste infiniment précieux ${ }^{2}$, le corps sorcier est appelé à retourner au néant. Il demeure que ce corps, il a bien fallu l'imaginer, le construire intellectuellement et procéduralement avant de l'imposer sur la scène judiciaire pour mieux le détruire rituellement...

L'imaginer d'abord, dans la mesure où le corps sorcier participe de ce puissant imaginaire de la sorcellerie démoniaque et sectaire qui s'est constitué durant les premières décennies du $\mathrm{XV}^{\mathrm{e}}$ siècle dans un espace essentiellement alpin, du Dauphiné aux rives du lac Léman, de Lyon à Bâle, soit au cœur d'une Chrétienté qui se sait ou se croit menacée d'éclatement. Mais aussi le construire judiciairement, en ce sens que cet imaginaire se donne pour vrai et qu'il revient notamment aux diverses instances judiciaires impliquées dans la répression de la sorcellerie médiévale (l'Inquisition bien sûr, mais aussi certains tribunaux laïcs, municipaux ou royaux) de prouver la véracité des accusations portées contre les sorciers, de donner littéralement corps aux fantasmes des juges et plus généralement de cette «société de persécution» qui, au moins depuis le XIII ${ }^{\mathrm{e}}$ siècle, a pris conjointement ou successivement pour cibles les juifs, les lépreux, les hérétiques et enfin les sorciers et les sorcières ${ }^{3}$.

\footnotetext{
1 Jacques Du Clercq, Mémoires, éd. F. de Reiffenberg, Bruxelles, 1835-1836, livre IV, chap. 12 , p. 53.

${ }^{2}$ M.-C. Pouchelle, «Représentations du corps dans la Légende dorée », Ethnologie française, 6, 1976, $\mathrm{n}^{\circ}$ spécial , «Langages et images du corps », p. 293-308.

${ }^{3}$ R. I. Moore, La persécution. Sa formation en Europe ( $X^{e}-X I I I^{e}$ siècle), Paris, Les Belles Lettres, 1991 ( $1^{\text {re }}$ éd. angl. 1987) et C. Ginzburg, Le sabbat des sorcières, Paris, Gallimard, 1992 (1 $1^{\text {re }}$ éd. ital., 1989). Sur les débuts médiévaux de la chasse aux sorcières, voir la synthèse commode de J.-P. Boudet, «La Genèse médiévale de la chasse aux sorcières. Jalons en vue d'une relecture", dir. N. Nabert, Le Mal et le diable. Leurs figures à la fin du Moyen Âge, Paris, 1996, p. 34-52.
}

Cahiers de Recherches Médiévales, 13, 2006 
Mais si la justice s'acharne autant sur le corps $d u$ sorcier, c'est aussi que ce dernier ne forme que la partie d'un tout, le sorcier n'étant que le membre d'un corps collectif, le corps sorcier ou diabolique dont la matérialisation puis la destruction constituent l'une des visées principales de la chasse aux sorcières. L'originalité persécutrice de la sorcellerie démonolâtre (telle qu'elle se constitue dans la première moitié du $\mathrm{XV}^{\mathrm{e}}$ siècle) est de faire converger la multitude des actes dispersés et individualisés de magie noire ou blanche, populaire ou savante, dans un seul cadre angoissant et totalisant: celui d'un vaste complot contre la société chrétienne. La traditionnelle jeteuse de sort, le magicien de cour ne sont plus des figures isolées, détentrices d'une puissance magique ambivalente, alternativement guérisseuse ou meurtrière, mais les instruments humains d'un vaste complot ourdi par le diable. C'est bien en effet le poids du nombre, la puissance du collectif qui assure la transformation de la traditionnelle et sans doute immémoriale sorcellerie paysanne et/ou de la magie savante en une formidable et redoutable hérésie démoniaque. Dès le premier tiers du $\mathrm{XV}^{\mathrm{e}}$ siècle, une abondante littérature tout à la fois technique et doctrinale se développa, en étroit contact avec la pratique procédurale: le Formicarius de Jean Nider, les Errores gazariorum, le traité du juge dauphinois Claude Tholosan ${ }^{4}$ et plus tard autour de 1460, le Flagellum hareticorum fascinariorum $^{5}$ ou la Recollectio d'Arras ${ }^{6}$ constituent les jalons textuels les plus significatifs de ce puissant effort compilatoire, pratique et intellectuel pour appréhender le péril sorcier. On y retrouve une image de plus en plus précise des assemblées nocturnes des sectateurs du diable qui, bien avant le $\mathrm{XVI}^{\mathrm{e}}$ siècle, contribue à fixer définitivement le stéréotype du sabbat des sorciers et des sorcières.

En prenant pour cadre chronologique les décennies centrales du $\mathrm{XV}^{\mathrm{e}}$ siècle (1430-1470) et comme source principale les premiers textes théoriques et procéduraux relatifs au sabbat, nous voulons souligner la place du corps sorcier dans la répression, à la fois d'un point de vue individuel (le corps $d u$ sorcier) et collectif (le corps sorcier) dès lors que la hantise du complot sorcellaire qui se déploie à l'aube historique de la modernité nous apparaît révélateur de la crise concomitante d'un autre grand corps collectif, celui de la Chrétienté ${ }^{7}$. Ne peut-on voir alors dans l'emprise croissante de cet imaginaire redoutable sur les esprits (notamment de tous ceux qui s'estiment investis de la mission de préserver l'unité du peuple chrétien) les

\footnotetext{
${ }^{4}$ Jean Nider, Formicarius (vers 1437-1438), Anonyme, Errores gazariorum... (vers 1437), Claude Tholosan, Ut magorum et maleficiorum errores..., (vers 1436). Ces trois textes fondamentaux, et d'autres moins connus, se trouvent réunis dans le remarquable dossier composé par l'équipe des médiévistes de l'université de Lausanne rassemblée autour d'Agostino Paravicini-Bagliani, L'Imaginaire du sabbat. Edition critique des textes les plus anciens (1430 c.-1440 c.), réunis par M. Ostorero, A. Paravicini-Bagliani, K. Utz Tremp et C. Chêne, (Cahiers Lausannois d'histoire médiévale, n²6), Lausanne 1999.

${ }^{5}$ Nicolas Jacquier, Flagellum hareticorum fascinariorum, Francfort sur Main, 1581, édition partielle dans J. Hansen, Quellen und Untersuchungen zur Geschichte des Hexenwahns und der Hexenverfolgung im Mittelalter, Bonn, 1901, réimpr. Hildesheim, 1963, p. 133-145.

6 Anonyme, Recollectio casus, status et condicionis Valdensium ydolatrarum, édition ancienne du texte d'après le ms. lat. 3446 de la BnF, J. Hansen, op. cit., p. 149-183.

${ }^{7}$ Sur la richesse de la notion de corps au Moyen Âge, voir notamment J.-C. Schmitt, «Le corps en chrétienté », La production du corps. Approches anthropologiques et historiques, dir. M. Godelier et M. Panoff, Paris, éd. des archives contemporaines, 1998, p. 339-355.
} 
effets d'un profond désarroi lié à l'ébranlement de la Chrétienté au temps du grand schisme et des recompositions politiques autour de l'État moderne?

\section{Corps du délit, corps du délire}

Pour implanter la persécution des sorciers, il fallait d'abord faire admettre la réalité même du sabbat en démontrant que celui-ci, loin d'être un songe inoffensif, s'inscrivait dans une réalité physique tangible et pouvait donc faire l'objet d'une expérience sensible autant que d'une enquête judiciaire. Ce choix en faveur de la réalité empirique du sabbat impliquait de rompre avec une longue et vénérable tradition savante de scepticisme face aux récits de rencontres et de chevauchées nocturnes avec les démons. Cette tradition s'exprimait notamment dans le fameux Canon Episcopi, un texte capitulaire du $\mathrm{IX}^{\mathrm{e}}$ siècle qui reléguait ces récits au rang de fantasmagories ${ }^{8}$. Acteurs et théoriciens de la chasse aux sorcières (il s'agit souvent, mais pas toujours, des mêmes personnes) ont donc très tôt éprouvé la nécessité de convaincre leurs contemporains de la réalité physique des assemblées rituelles autour du diable 9 . À cet égard, le corps des sorciers joue un rôle important dès lors qu'il s'agit justement (dans cette phase pionnière et militante de la persécution antisorcière) de prouver l'ancrage matériel du sabbat.

Il existe à ce propos un texte très révélateur de l'importance reconnue au corps du sorcier dans la démonstration de l'existence physique du sabbat. Il s'agit $d u$ Flagellum hareticorum fascinariorum, traité composé en Flandres vers 1458 par l'inquisiteur dominicain Nicolas Jacquier ${ }^{10}$. Dans la perspective polémique qui est la sienne, N. Jacquier se sert du corps pour démontrer la réalité d'un danger que d'aucuns persistent à situer du côté de l'illusion. C'est que le rôle de sectateur du diable, selon Jacquier, n'est pas de tout repos. De leur participation aux fêtes diaboliques, les sorciers conservent des séquelles physiques qui les obligent à garder le lit plusieurs jours ${ }^{11}$. Ces courbatures consécutives aux débauches de la nuit confirment bien, selon lui, la réalité matérielle du sabbat. Pour un inquisiteur comme Jacquier, très soucieux de prouver la réalité physique du sabbat contre les tenants du Canon Episcopi, le corps des prétendus sorciers joue un rôle important dans sa démonstration comme support et preuve physiologique de l'existence du sabbat. La preuve par les courbatures doit cependant être replacée dans le cadre d'une stratégie argumentative globale qui vise moins à démontrer devant un tribunal la culpabilité des accusés que le caractère réaliste du sabbat. Car il ne s'agit pas d'une preuve au sens judiciaire du terme mais plutôt d'un argument parmi d'autres en faveur d'une

${ }^{8}$ Faussement attribué à un concile d'Ancyre du $\mathrm{IV}^{\mathrm{e}}$ siècle, le texte entra finalement au XII ${ }^{\mathrm{e}}$ siècle dans le Décret de Gratien, ce qui acheva de lui conférer une autorité presque irréfragable: Gratien, Decretum, Pars II, Causa XXVI, Quæstio V, c. 12 (éd. Friedberg I, col. 1030-1031).

9 Commode synthèse de F. Cardini, Magia, stregoneria, superstizioni nell'Occidente medievale, Florence, La Nuova Italia Editrice, 1979, p. 81-85.

${ }^{10} \mathrm{~N}$. Jacquier, op. cit.

${ }^{11}$ N. Jacquier, op. cit., éd. J. Hansen, 1901, p. 136: «quod plures ex eis inde postea per aliquot dies manent afflicti et debilitati... ». 
position doctrinale. Du reste, la preuve par le corps trouve assez rapidement ses limites dans un contexte plus spécifiquement judiciaire.

Il ne semble pas ainsi que l'on ait recherché dès ce moment sur le corps de l'accusé la fameuse marque de la sorcière, cette zone corporelle réputée insensible à la douleur, signe de l'appartenance au diable ${ }^{12}$. Du moins, les notations à cet égard sont encore très floues. Dans l'un des premiers textes à décrire précisément le rituel du sabbat (connu sous le nom des Errores Gazariorum), l'auteur anonyme fait bien état d'un pacte rédigé avec le sang de la main gauche de l'impétrant, mais sans tirer davantage de conséquences quant à la possible rémanence de l'opération sur la main coupable $^{13} \ldots$ Il faut attendre le milieu du $\mathrm{XV}^{\mathrm{e}}$ siècle (et notamment le traité de N. Jacquier) pour voir le motif de la marque se préciser véritablement. Ce dernier rapporte en effet le cas d'un homme qui confesse avoir été consacré au diable dès son plus jeune âge: sa mère l'ayant conduit à la synagogue pour le présenter au diable (avec ses frères et sœurs), il est accueilli par un démon en forme de bouc qui lui imprime (impressit) sur la cuisse une marque (signum) en forme de fève ${ }^{14}$. Il s'agit là sans doute de la mention la plus précoce et la plus précise de la marque qui, comme on le voit, intervient dans un contexte rituel inspiré directement du baptême chrétien ${ }^{15}$. Dans la perspective polémique qui est la sienne, le corps a donc encore son mot à dire; le langage du corps fait sens. Néanmoins, cette idée ne trouve pas encore d'application judiciaire. Rien ne prouve ainsi que les juges d'Arras, au moment de la célèbre Vauderie de 1459-1460, aient recherché cette marque de possession sur le corps des accusés à l'aide d'une aiguille ou de tout autre instrument pointu. Tout juste se servent-ils devant la foule, lors du cérémonial de condamnation, de certaines particularités du corps des accusés : l'un d'eux fut invité par l'inquisiteur à exhiber son pouce mutilé comme preuve matérielle de ce qu'il avait conclu dans le sang un pacte avec le diable ${ }^{16}$. La marque apparaît encore en creux et les juges ne la recherchent pas encore frénétiquement sur le corps du sorcier. Il est vrai que Jacques du Bois, l'auteur présumé de la Recollectio (qui consigne l'expérience procédurale du tribunal d'Arras ${ }^{17}$ ) recommande aux juges de

\footnotetext{
${ }^{12}$ Sur la marque de la sorcière, l'étude fondamentale reste celle de F. Delpech, «La marque des sorcières : logique(s) de la stigmatisation diabolique », Le sabbat des sorciers en Europe $\left(X V^{e}-X V I I I^{e}\right.$ siècles), dir. N. Jacques-Chaquin et M. Préaud, Grenoble, 1993 (Colloque international E.N.S. Fontenay-Saint-Cloud, 4-7 novembre 1992), p. 347-368. Tout aussi essentielle, la mise au point récente de M. Ostorero, «Les marques du diable sur le corps des sorcières $\left(\mathrm{XIV}^{\mathrm{e}}-\mathrm{XVII}{ }^{\mathrm{e}}\right.$ siècles)», La pelle umana, Micrologus (Natura, Scienze e Società Medievali), XIII, 2005, p. 359-388.

${ }^{13}$ Errores, op. cit., p. 296-297.

${ }^{14}$ J. Hansen, op. cit., p. 137.

${ }^{15}$ M. Ostorero, op. cit., p. 380.

${ }^{16}$ «Icellui Jehan du Bois, lui estant sur le hourt, l'inquisiteur lui demanda s'il n'estoit point vrai qu'il euist donné au diable, quant il lui fit hommaige, l'ongle de son pouce; lequel respondit publiquement que oui, et monstra son pouce devant tout le peuple. », J. Du Clercq, Mémoires, op. cit., livre IV, chap. 8, p. 39.

${ }^{17}$ Sur l'attribution de la Recollectio à Jacques du Bois, gradué de l'Université de Paris et doyen de la cathédrale d'Arras, voir F. Mercier, La Vauderie d'Arras. Une chasse aux sorcières à l'Automne du Moyen Âge, Rennes, P.u.R., 2006, spécialement p. 31-32.
} 
dévêtir les suspects avant de les soumettre à l'interrogatoire ${ }^{18}$. Il s'agit alors d'examiner attentivement leur corps afin de repérer d'éventuelles mutilations physiques (ongle arraché, etc.) en «signe du pacte» (signum pacti) ou bien de leur confisquer tout objet (comme un anneau ou un morceau de fil) susceptible de les immuniser contre la douleur. Mais le signum des vaudois-sorciers d'Arras correspond moins à une marque précise inscrite sur le corps des sorciers qu'à une lacune, qu'à une mutilation volontaire opérée par le nouvel adepte (ablation du pouce, d'un fragment du corps) pour sceller le pacte diabolique.

Plus que sur le corps de l'accusé, c'est sur sa parole, sur l'aveu que comptent les jugent pour parvenir à la vérité. Il s'agit moins en effet de lire la vérité sur le corps des accusés (sous la forme de la marque ou même de simples courbatures !) que de l'arracher de l'intérieur du corps sous la forme de l'aveu ${ }^{19} . .$.

À l'évidence, le seul langage du corps ne suffit pas à établir la preuve en justice. Si les réactions physiques des accusés (pâleur excessive, rougissements, etc.) sont effectivement prises en considération dans le cadre de l'interrogatoire, elles n'ont cependant qu'une faible valeur probatoire. La preuve matérielle par le corps est insuffisante et c'est d'abord à travers l'aveu, par la parole contrainte fixée par écrit, que se construit et s'impose la «vérité » judiciaire du sabbat.

Dans cette nouvelle perspective procédurale, le corps fait désormais obstacle à la vérité en tant qu'il résiste à l'épreuve de la torture. Dans la Recollectio, la pratique de la torture s'apparente ainsi à une séance d'exorcisme où les organes de la parole du prévenu sont physiquement neutralisés par le démon pour empêcher l'accusé de dévoiler le contenu du sabbat. Il faut briser les corps par le moyen de la torture pour «libérer» la parole. L'interrogatoire se transforme en un «combat» (certamen) qui oblige le juge à affronter le diable pour lui faire lâcher prise et arracher ainsi l'aveu ${ }^{20}$.

Il reste que cette patiente et laborieuse reconstitution «de ce qui s'est vraiment passé » accorde une place stratégique au corps, non pas seulement parce que celui-ci est soumis à la torture (et que la douleur physique délie les langues), mais aussi parce que les juges sollicitent les prétendues sensations du corps au sabbat.

L'assemblée nocturne des sorciers est le lieu du secret par excellence. Non seulement les réunions sont supposées se dérouler au cœur de la nuit, en des lieux solitaires, mais les démons eux-mêmes en protègent les accès contre d'éventuels intrus. Un petit traité anonyme composé dans la région de Lyon vers 1450 soutient ainsi, après d'autres, que le diable assure la confidentialité des rencontres à l'aide de puissants sortilèges d'invisibilité, permettant ainsi aux sorciers de voir sans être vus :

\footnotetext{
${ }^{18}$ J. Hansen, op. cit., p. 155.

${ }^{19}$ Conformément à la logique de la procédure inquisitoire (de type extraordinaire) qui prévaut généralement dans les procès de sorcellerie du $\mathrm{XV}^{\mathrm{e}}$ siècle, dès lors du moins que la sorcellerie est assimilée au crime de majesté. Voir notamment, J. Chiffoleau, «Sur le crime de Majesté médiéval ", Genèse de l'État moderne en Méditerranée, Rome, éd. École française de Rome, 1993, p. 183-213 et Y. Thomas, «'Arracher la vérité'. La Majesté et l'Inquisition $\left(\mathrm{I}^{\mathrm{er}}-\mathrm{IV}^{\mathrm{e}}\right.$ siècles ap. J.-C. », Le Juge et le jugement dans les traditions juridiques européennes, dir. R. Jacob, Droit et société, vol. 17, 1996, p. 15-41.

${ }^{20}$ Jacques du Bois, Recollectio, op. cit., p. 168.
} 
De même, ladite synagogue diabolique se tient communément en un lieu toujours séparé, éloigné et écarté de toute affluence humaine. Et surtout, elle se tient aux carrefours, comme ils disent, ou bien en des lieux où convergent de nombreuses routes. Cependant, ils ne peuvent pas être vus des autres voyageurs, comme ils disent, mais ils voient clairement ces voyageurs ${ }^{21}$.

En conséquence, engager la lutte judiciaire contre la sorcellerie démonolâtre consiste d'abord à percer l'occulte ${ }^{22}$. Et dans cette perspective, le corps des sorciers présumés constitue l'une des premières clés d'accès à l'occulte diabolique. Puisque le diable dresse des écrans, des rideaux de fumée autour des activités de la secte, c'est en sollicitant le témoignage intérieur (le point de vue interne!) des participants que le juge prétend découvrir et reconstituer le déroulement du cérémonial sabbatique. En l'absence du témoignage classique (externe), l'enquête judiciaire vise donc à explorer le contenu des assemblées par l'entremise exclusive de ses participants. La force de l'enquête réside en effet dans la certitude qu'ont les juges de pouvoir atteindre la vérité du sabbat à travers le témoignage des accusés. Dans un passage décisif de son traité consacré à l'art et la façon d'interroger les suspects de sorcellerie, Jacques du Bois expose les différents modes de connaissances possibles du sabbat par le juge. "C'est en effet par le moyen de quatre sens - par un sens intérieur (à savoir par la force cogitative ou estimative) et par trois sens extérieurs (à savoir par la vision, par l'ouïe et le toucher principalement lors des étreintes et du coït) et même parfois par la senteur et l'odorat... ${ }^{23} »$ que les participants au sabbat peuvent distinguer les hommes des démons. La connaissance par la vision est réputée certaine «car très souvent le démon a de gros yeux et, lors de ces assemblées, il a toujours des yeux étonnants, terribles, durs, brûlants, enflammés, brillants, scintillants, etc.». Le texte dessine ainsi les étapes d'une démarche cognitive conforme aux données de la culture savante. Selon les représentations anthropologiques les plus communes à cette époque, la personne humaine n'est pas seulement un corps mais résulte de l'assemblage du corps et de l'âme. Il faut notamment que l'âme ait un corps pour connaître et appréhender le monde extérieur. Le corps permet, autant que l'âme du pécheur, de connaître la vérité. Le juge est ainsi appelé à franchir les différents degrés de la connaissance sensible qui mobilise plusieurs puissances de l'âme, de la puissance estimative (comparable finalement à l'instinct) à la puissance sensitive (fondée sur les cinq sens). Il va de soi que derrière ce genre de discours très théorique (littéralement démarqué de la théorie thomiste de la connaissance ${ }^{24}$ ) se profile une réalité procédurale très concrète, puisque la théorie inspire le questionnaire que les juges utilisent lors des interrogatoires... La richesse des descriptions du diable au sabbat, par accumulation d'adjectifs, trouve ici une partie de sa raison d'être. La volonté des juges d'ancrer le crime supposé des

\footnotetext{
${ }^{21}$ Anonyme, La Vauderye de Lyonois en brief, J. Hansen, op. cit., p. 190, trad. du latin. Une nouvelle édition critique de ce texte est en cours en collaboration avec M. Ostorero.

${ }^{22}$ Voir notamment J. Chiffoleau, «Dire l'indicible. Remarques sur la catégorie du nefandum

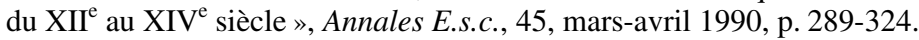

${ }^{23}$ Jacques du Bois, Recollectio, op. cit., p. 174-175.

${ }^{24}$ É. Gilson, Le Thomisme, Paris, Vrin, 1965, p. 258-262.
} 
sorciers dans la réalité la plus matérielle possible induit un questionnaire d'enquête attentif à la description des entités matérielles, physiques ou spirituelles rencontrées au sabbat. De sorte que juges et accusés finissent parfois par communier dans le même délire.

Il faut croire que l'influence de ce type de questionnaire était considérable puisque certains accusés en arrivent pour ainsi dire à voir le diable, à le toucher même, sans l'avoir jamais rencontré réellement. Et l'on peut mesurer la puissance suggestive, voire auto-suggestive, de ce type de questionnaire dans le premier procès de Jeanne d'Arc (celui de 1431) lorsque celle-ci est amenée, sous le feu croisé des questions très précises de ses juges, à voir progressivement ce qu'elle ne faisait qu'entendre au début de son procès.

Capturée le 24 mai 1430 devant Compiègne, Jeanne d'Arc fut remise contre rançon entre les mains des Anglais qui la traduisirent à Rouen devant un tribunal ecclésiastique mixte associant l'évêque de Beauvais, Pierre Cauchon, et le viceinquisiteur de France, Jean Le Maistre. La Pucelle ne fut pas exactement accusée de se rendre au sabbat et ses juges écartèrent explicitement l'hypothèse qu'elle pût être une «sorcière vaudoise». Mais les savantes grilles d'analyse appliquées à son cas particulier n'en sont pas moins sous-tendues par les mêmes références démonologiques, les mêmes inquiétudes aussi qui, ailleurs, dans un autre contexte mais simultanément, se cristallisent dans l'imaginaire du sabbat. Si Jeanne d'Arc ne correspond pas exactement au stéréotype de la sorcière démonolâtre (tel qu'il s'impose dans les années centrales du $X^{\mathrm{e}}$ siècle), elle n'en est pas moins considérée par ses juges comme une magicienne et une hérétique en proie à des illusions diaboliques ${ }^{25}$. D'où l'importance que les juges accordent aux aspects étranges ou merveilleux de son parcours politique et militaire exceptionnel, et notamment, comme on sait, aux «voix » dont elle prétend, jusque dans sa prison de Rouen, recevoir conseil et soutien. Entre le 21 février 1431 et la fin du mois de mars de la même année, Jeanne d'Arc subit une série d'interrogatoires devant différents auditoires issus de ce tribunal mixte. L'analyse chronologique de ses réponses manifeste l'évolution sensible de son témoignage relatif à ses « voix ».

Au début de son procès, Jeanne évoque surtout l'action persuasive d'une ou de plusieurs «voix» dont la manifestation s'associe à des phénomènes visuels encore relativement imprécis, comme cette "grande clarté» signalée dans son interrogatoire du 22 février ${ }^{26}$. D'abord très évasive sur l'origine et la nature exacte de ses voix, Jeanne est progressivement amenée, sous l'influence des juges qui la pressent de questions, à leur donner une apparence de plus en plus précise. Tout se passe comme si, au fil des interrogatoires, la Pucelle en venait elle-même à voir ce qui n'était au départ qu'un phénomène d'ordre essentiellement auditif :

Interrogée: comment sait-elle que l'apparition est homme ou femme?

Elle répondit qu'elle le sait bien et les reconnaît à leur voix et qu'elles le lui ont révélé; elle ne sait rien que ce ne soit par révélation et commandement de Dieu. Interrogée: quelle aspect leur voit-elle?

${ }^{25}$ C. Beaune, Jeanne d'Arc, Paris, éd. Perrin, 2004, p. 298-367.

${ }^{26}$ Procès de condamnation de Jeanne d'Arc, éd. et trad. de P. Tisset et Y. Lanhers, Paris, Société de l'histoire de France, éd. Klincksiek, 1960-1970, t.2, p. 46-47. 
Elle répondit qu'elle voit leur visage.

Interrogée si les saintes qui lui apparaissent ont des cheveux:

Elle répondit: «C'est bon à savoir».

Interrogée s'il y avait quelque chose entre leurs couronnes et leurs cheveux :

Elle répondit que non.

Interrogée si leurs cheveux étaient longs et pendants:

Elle répondit: «Je n'en sais rien». Elle dit aussi qu'elle ne sait pas si elles avaient quelque chose comme des bras ou si elles avaient d'autres membres figurés. Item elle dit qu'elles parlaient très bien et à merveille et elle les comprenait très bien. Interrogée: comment parlaient-elles alors qu'elles n'avaient pas de membres?

Elle répondit: «Je m'en rapporte à Dieu »; Item elle dit que la voix est douce, belle, humble et parle la langue française ${ }^{27}$.

L'interrogatoire du $1^{\mathrm{er}}$ mars est marqué par une tentative désespérée pour revenir encore et toujours à la voix ou les voix dont elle prétend tirer son inspiration. Parallèlement, on perçoit très bien comment ses juges s'efforcent de l'attirer du côté de la corporéité qui est aussi, en l'occurrence, celui du diabolique. Et de ce fait, sans qu'il y eût besoin de recourir à la torture physique, l'inspiration mystique de Jeanne, aussi vague fût-elle initialement, revêtit peu à peu la forme d'apparitions corporelles très concrètes (avec cheveux, tête, bras, jambes...), qui le refoulaient toujours davantage du côté de la démonolâtrie. Les incertitudes de Jeanne, les contraintes de l'interrogatoire, ainsi que le questionnement très technique des juges, permirent aux voix et aux premières visions de la Pucelle d'accroître leur consistance d'une façon spectaculaire. À la fin de ses interrogatoires, Jeanne d'Arc ne s'était pas encore complètement détachée des représentations lumineuses et évanescentes de ses voix :

Interrogée si, quand les saintes viennent à elle, il y a une lumière avec elles et si elle n'a point vu de lumière la fois où elle entendit la voix dans le château, ne sachant si elle était dans sa chambre. Elle répondit qu'il n'est point de jour où elles ne viennent en ce château et elles ne viennent pas sans lumière. Et pour cette fois sur laquelle on l'interroge, elle ne se souvient pas si elle a vu une lumière ni même si elle a vu sainte Catherine ${ }^{28}$.

Toutefois, le processus de matérialisation était déjà bien avancé. Suffisamment en tous cas pour que ses juges puissent en définitive l'incriminer et la condamner, entre autres, sous le chef de magie maléfique et d'idolâtrie.

Le procès de Jeanne d'Arc, comme d'autres procès apparentés de sorcellerie, rendent ainsi compte d'une autre obsession fondamentale des doctes : celle d'une sorte d'incarnation démoniaque... Au point qu'il est permis de se demander si la chasse aux sorciers et aux sorcières ne revient pas aussi à invoquer (engendrer ?) un autre corps collectif rival de celui de l'Église, pour mieux ensuite le détruire dans un corps à corps terrible entre le bien et le mal.

${ }^{27}$ Château de Rouen, jeudi $1^{\mathrm{er}}$ mars, cinquième séance publique, op. cit., p. 84-85.

${ }^{28}$ Château de Rouen, quatrième séance d'interrogatoire du mercredi 14 mars, en prison, op. cit., p. 128. 


\section{S'incorporer au diable?}

Il est tentant de considérer le sabbat comme le lieu fantasmatique d'une exaltation débridée de la chair et de ses plaisirs, en contraste saisissant avec l'idéal d'ascétisme et de mortification prôné par un certain christianisme des élites. Il est vrai que l'une des principales motivations des candidats à l'initiation diabolique serait, selon les premiers textes sur le sabbat des sorcières, la lubricité, la quête effrénée du plaisir sexuel. Pour l'auteur anonyme des Errores Gazariorum, les hommes et les femmes qui succombent aux attraits de la secte «désirent plus que tout se vautrer lubriquement dans l'acte charnel et (...) là ils satisfont à satiété leurs dispositions lubriques ${ }^{29} »$. Mieux encore, le diable glisse parfois dans la nourriture servie au sabbat des substances euphorisantes ou excitantes destinées à accroître les sensations des participants. En ce sens, la description des agapes diaboliques n'est pas sans rappeler, sous une forme diabolisée, certaines pratiques festives de la jeunesse paysanne ou citadine. Le fait est que les théologiens ou les prédicateurs ont pu se servir du sabbat comme d'un instrument de culpabilisation collective contre certaines formes de divertissement populaire jugées contraire à l'ordre moral ${ }^{30}$. Dans le contexte de la Vauderie d'Arras, la première cible de la persécution correspond à la prostitution urbaine qui avait alors pignon sur rue. Dès lors, aucune solution de continuité n'existe entre la fréquentation des prostituées et celle du sabbat. Du bordel municipal au sabbat, il n'y a parfois qu'un saut à faire que l'utilisation du balai magique permet aisément d'accomplir, comme dans cette évocation peu connue du sabbat conservée dans les archives du Parlement de Paris...

Lequel, après serment solempnellement fait, dit et confessa sans induction, menaces ne tortures aucunes, que depuis trois ans en ça, il avoit eu congnoissance a une femme d'Arras nommée la Pigneresse et que a une fois ils conclurent de coucher ensemble. Et dit que ladite Pigneresse lui demanda s'il vouloit venir a une belle compaignie, qui dit que oy et apres qu'il eurent descendus les degrés de l'ostel, ladite Pigneresse oygnit un baston d'oing noir sur lequel ils monterent et tantost apres se trouverent au bois de Moflaines ou trouverent plusieurs personnes assises a table dont le premier estoit en espèce de cinge roux, et avoient de plusieurs viandes ${ }^{31} \ldots$

Il apparaît donc légitime sans doute de voir dans le mythe sabbatique une déformation caricaturale et hostile de divertissements réels, cibles privilégiées d'une réforme moralisatrice véhiculée par une prédication agressive ${ }^{32}$. Pour autant, il convient de souligner que l'exaltation apparente de la chair au sabbat s'accompagne d'une hantise de la stérilité : celle-ci affecte les corps des sorciers eux-mêmes puisque les amours diaboliques sont par essence contre-nature, c'est-à-dire

${ }^{29}$ Errores gazariorum, op. cit., p. 296-297.

${ }^{30}$ R. Muchembled, «L'Autre côté du miroir: mythes sataniques et réalités culturelles aux $\mathrm{XVI}^{\mathrm{e}}$ et XVII ${ }^{\mathrm{e}}$ siècles », Annales ESC, mars-avril 1985, n ${ }^{\circ}$ 2, p. 288-306, spécialement p. 296.

${ }^{31}$ Audience du 21 mai 1461, A. N., X/2a/28, fol. 381v. Il s'agit d'un extrait de la plaidoirie de Guillaume de Ganay pour le compte des vicaires d'Arras, défendeurs dans le procès.

${ }^{32}$ J. Rossiaud, La prostitution médiévale, Paris, Flammarion, 1988 (réed. coll. «champsflammarion », 1990), spécialement p. 169. 
détournées des seules fonctions légitimes de la procréation ${ }^{33}$. Par ailleurs, on sait bien que l'une des principales activités de la secte consiste justement à frapper de stérilité tout ce et ceux qu'elle touche avec ses poudres et ses sortilèges.

Bien plus, l'image de la fusion des corps au sabbat, dans l'orgie sexuelle à rebours de la «nature», exprime ainsi sans doute une autre inquiétude plus fondamentale : celle de la formation, dans les replis de la clandestinité, d'un corps collectif qui se nourrit et grandit aux dépens d'un autre corps affaibli dans ses fonctions vitales, celui de la société chrétienne. L'exaltation de la chair au sabbat va en effet de pair avec le sentiment d'un dépérissement du corps social légitime. Il faut bien voir en effet que le rituel d'initiation soigneusement décrit par les textes se présente d'abord comme un rituel d'incorporation. Ou plus exactement, le mythe du sabbat offre, dès la première moitié du $\mathrm{XV}^{\mathrm{e}}$ siècle, un lieu qui permet de penser à la fois la dissolution d'un lien fondamental préexistant (celui qui unit le fidèle à la communauté de l'Église) et sa recomposition, presque simultanée, autour d'une figure antithétique de celle du Christ, le diable. C'est ainsi que plusieurs traités démonologiques font le choix de présenter les modalités de l'entrée dans la secte à travers l'exemple d'un seul postulant (masculin ou féminin) que le lecteur est invité à suivre à travers toutes les étapes de son parcours initiatique, depuis sa prise de contact avec les sorciers jusqu'à l'adoration du diable ${ }^{34}$. Cet individu-témoin, après avoir rendu hommage au diable, finit cependant toujours par disparaitre, par se fondre dans le groupe pour ne plus jamais réapparaître dans le récit. La puissance du collectif finit toujours par se substituer à l'expérience singulière. De ce point de vue, il est permis de se demander si la mêlée indistincte des corps dans le défoulement sexuel qui accompagne et clôt nécessairement la réunion démoniaque des sorciers et des sorcières ne traduit pas aussi, sur un mode paroxystique, cette volonté de faire corps qui traverse l'imaginaire du sabbat. D'où peut-être aussi l'importance donnée à un thème comme celui de la profanation de l'hostie, du corpus verum du Christ.

La profanation de l'hostie (corpus christi) constitue l'un des motifs récurrents de l'image savante du sabbat. Certes, son importance relative peut varier d'un auteur à l'autre selon que ce dernier est plus (Claude Tholosan, Jean Taincture ou Tinctor ${ }^{35}$ ) ou moins (l'Anonyme des Errores) sensible à cet aspect du sabbat, mais celui-ci reste incontournable. L'hostie fait ainsi l'objet, sous des formes diverses, d'une agression ritualisée systématique lors de la réception des nouvelles recrues. Par ailleurs, le diable lui-même en fait paradoxalement si grand usage (pour accomplir les sacrilèges) qu'il recommande souvent à ses adeptes d'en dérober sur les autels consacrés. Chez certains auteurs, l'hostie entre même dans la composition des onguents dont les sorciers se servent pour accomplir leurs forfaits ou leurs exploits les plus extravagants (comme le vol magique). À parcourir cette première littérature savante sur le sabbat, on est ainsi frappé de l'importance accordée à la thématique de la profanation de l'hostie et, par delà le corps sacramentel du Christ, à l'agressivité

\footnotetext{
${ }^{33}$ Sur la catégorie juridique du contre-nature et ses dimensions politiques, J. Chiffoleau, "Contra Naturam. Pour une approche casuistique et procédurale de la nature médiévale», Micrologus, $\mathrm{n}^{\circ}$ 4, «Il teatro della natura », Lausanne, 1996, p. 265-312.

${ }^{34}$ C'est le cas des Errores Gazariorum comme, plus tard, de la Recollectio d'Arras.

${ }_{35}$ Jean Tinctor, Invectives contre la secte de vauderie, éd. E. Van Balberghe et F. Duval, Tournai-Louvain-La-Neuve, 1999.
} 
foncière de la sorcellerie diabolique à l'encontre de l'incarnation. Certains auteurs, comme par exemple Claude Tholosan, le déclarent sans ambage: «il faut savoir aussi que Satan, cet ange apostat, s'efforce principalement d'agir dans cette secte exécrable à l'encontre de ce qui se rapporte à l'humanité de $\operatorname{Dieu}^{36} \ldots$ ». Conformément à la logique d'inversion qui commande la structure du sabbat ${ }^{37}$, l'importance de la profanation de l'hostie est à la mesure de son exaltation parallèle dans la «vraie»vie spirituelle et liturgique ${ }^{38}$. Une étroite et étrange proximité unit l'essor considérable du culte de l'eucharistie à la fin du Moyen Âge et la construction du sabbat. On sait par exemple qu'il en faut très peu pour que les célestes et pures visions du Christ souffrant valorisées par la dévotion moderne ne basculent soudain du côté diabolique ${ }^{39}$. De même, les plus minutieuses descriptions des pratiques diaboliques enregistrent parfois, avec la sensibilité d'un sismographe, les moindres fluctations des débats théologiques et liturgiques contemporains autour de l'eucharistie ${ }^{40}$. Que les vaudois-sorciers consomment en effet du pain et du vin (fut-il tourné en vinaigre) lors des banquets offerts par le diable n'est pas sans rapport sans doute avec les controverses du moment autour de la communion sous les deux espèces (revendiquée notamment par les hussites).

À cet égard, le mythe sabbatique apparaît bien comme le miroir déformé et angoissé d'un christianisme de la peur dont certaines élites socio-culturelles sont porteuses. Il est aussi et peut-être surtout l'expression d'une inquiétude plus fondamentale liée à la déliquescence d'un autre corps collectif dont l'eucharistie constitue précisément la matrice référentielle ${ }^{41}$ : l'Église comme communauté atemporelle de tous les fidèles du Christ. Dans ces conditions, les attaques sacrilèges prêtées aux sorciers contre l'incarnation ne seraient pas seulement le révélateur d'un éventuel clivage culturel ou dogmatique entre les élites et le peuple, entre un christianisme intellectuellement élaboré (recentré autour du mystère de l'incarnation) et un christianisme populaire plus sommaire ${ }^{42}$. Elles seraient aussi bien l'envers cauchemardesque du rêve unitaire qui hante la Chrétienté au moment même où celle-ci, sur fond de schisme et de contestation de la monarchie pontificale, commence précisément à se déliter comme espace théologico-politique fondamental.

En ce sens, la conception de la sorcellerie médiévale se situe bien, pour une part, dans le prolongement de l'hérésiologie traditionnelle: les sorciers, sectateurs

\footnotetext{
${ }^{36}$ Claude Tholosan, Ut magorum, op. cit., p. 376-377.

${ }^{37}$ Bien mise en évidence notamment par P. Paravy, «À propos de la genèse médiévale des chasses aux sorcières : le traité de Claude Tholosan, juge dauphinois (vers 1436) », Mélanges de l'École française de Rome, Moyen Âge - Temps modernes, 91, 1979, p. 333-379.

${ }^{38}$ M. Rubin, Corpus Christi. The Eucharist in Late Medieval Culture, Cambridge University Press, 1991.

${ }^{39} \mathrm{~J}$. Wirth, L'Image médiévale. Naissance et développements ( $V I^{e}-X V^{e}$ siècle) Paris, éd. Klincksieck, 1989, p. 338-340.

40 Voir les remarques suggestives d'E. Anheim, «Rituels sabbatiques et contestation évangélique, vers $1430 »$, Médiévales, 42, printemps 2002, p. 167-175.

${ }^{41}$ P. Von Moos, «l'individu ou les limites de l'institution ecclésiale », L'Individu au Moyen Âge, dir. B. Bezos-Rezac, D. Iognat-Prat, Paris, éd. Aubier, 2005, p. 271-288.

${ }^{42}$ Un aspect qu'il importe aussi de prendre en compte selon P. Paravy, «Le Traité de Claude Tholosan, juge dauphinois (vers 1436)», L'Imaginaire, op. cit., p. 417-438, spécialement p. 433-434.
} 
du diable, sont aussi parties prenantes d'un corps diabolique plus vaste dont le diable est la tête et eux les membres (membra diaboli) ${ }^{43}$. Dans sa Somme théologique, Thomas d'Aquin s'efforçait déjà, au XIII ${ }^{\mathrm{e}}$ siècle, de penser l'existence d'une sorte de royaume du mal opposé symétriquement au corps mystique du Christ ${ }^{44}$. Réfléchissant sur le statut du Christ comme tête de l'Église, le docteur évangélique en arrivait à s'interroger en effet sur la nature de l'emprise que le diable (rapproché de l'Antéchrist) pouvait exercer sur les hommes éloignés de Dieu. À la question de savoir si le diable pouvait se trouver à la «tête de tous les méchants », Thomas d'Aquin répondait d'abord par l'affirmative en reconnaissant l'aptitude du prince des démons à réunir sous son sceptre tous les hommes pervers. Il précisait bien toutefois que ce maléfique souverain n'exerçait sur les membres de son corpsroyaume qu'une contrainte extérieure qui n'affectait pas leur libre-arbitre. L'unité de cet étrange corps collectif du diable (pensé dans le miroir du légitime corps mystique du Christ ${ }^{45}$ ) reposerait donc sur l'adhésion volontaire de ses membres. C'est encore assez largement dans ce cadre de pensée tout à la fois technique, doctrinal et eschatologique que textes et images relatifs au sabbat se situent vers le milieu du $\mathrm{XV}^{\mathrm{e}}$ siècle ${ }^{46}$. On voit par là que le corps ne s'engage pas seul au sabbat, mais que c'est bien toute la personne (prise à la fois comme corps et âme, à travers l'exercice de la volonté et de l'intention) qui s'y trouve impliquée. La plupart des textes insistent bien sur ce point: les sorciers participent aux réunions nocturnes à l'état de veille (vigilanter), en pleine possession de leurs moyens physiques et intellectuels ${ }^{47}$.

En prenant le parti du mal, les sorciers contribuent ainsi à fortifier une entité collective dont les structures reproduisent celles de l'Église considérée comme le «corpus mysticum» du Christ. Voici probablement l'une des raisons pour lesquelles la question du corps reste au centre de l'image du sabbat qui apparaît comme le lieu symbolique, fantasmatique où tente de se réaliser une pseudo-incarnation du diable. Il ne s'agit bien en effet toujours que d'une tentative avortée, nécessairement approximative et imparfaite, ne serait-ce justement que parce que le diable, selon les

${ }^{43}$ Voir, par exemple, l'application de ce terme aux sorciers d'Arras par le moine Corneille de Zantfliet, Chronicon, E. Martène et V. Durand (éd.), Veterum scriptorum et monumentorum historicorum (Amplissima Collectio), Paris, 1724-1733, t. 5, p. 501-502. Sur son usage contre les hérétiques médiévaux, voir notamment G. G. Merlo, « Membra diaboli : demoni ed eretici medievali », Nuova rivista storica, sept.-déc. 1988, fasc. V-VI, p. 583-598.

${ }^{44}$ Thomas d'Aquin, Summa Theologia, III, q. 8, art. 7-8. Cf. id., Opera omnia, F. Frommann, V.G. Holzboog, Stuttgart, 1980, t. 2, p. 783-785.

${ }^{45}$ Sur cette notion théologico-politique essentielle, l'étude pionnière reste celle d'H. de Lubac, Corpus mysticum. L'Eucharistie et l'Église au Moyen Âge, Paris, 1944, suivie de celle d'E. Kantorowicz, Les Deux corps du roi. Essai sur la théologie politique au Moyen Âge, Paris, Gallimard, 1989, ( 1 éd. angl., 1957). Voir aussi J. Moingt, «Polymorphisme du corps du Christ », Le Temps de la réflexion, VII, Corps des Dieux, 1986, p. 47-61.

${ }^{46}$ Sur la première imagerie du sabbat des sorcières en lien avec le culte eucharistique, voir F. Mercier, «Un trompe-l'œil maléfique : l'image du sabbat dans les manuscrits enluminés de la cour de Bourgogne », Médiévales n44, printemps 2003, p. 97-116.

${ }_{47}$ Voir, par exemple, Nicolas Jacquier, Flagellum, op. cit., p. 136: «manifestum est quod huiusmodi apparitiones fiunt realiter, non a somniantibus sed a vigilantibus... ». 
données de la démonologie savante, ne possède pas de corps à proprement parler ${ }^{48}$. D'une certaine façon, il est permis de se demander si, à travers le mythe du sabbat, il ne s'agit pas néanmoins de faire advenir un corps sorcier diabolique, double caricatural et imparfait du corps christique pour mieux, ensuite, l'écraser et le détruire. Cette destruction devant nécessairement manifester le triomphe du seul corps légitime qui vaille, celui de l'Église, mais aussi sans doute celui de l'État territorial qui, tout en s'appliquant à lui-même depuis fort longtemps la métaphore organiciste ${ }^{49}$, commence au $\mathrm{XV}^{\mathrm{e}}$ siècle à prendre le relais de l'Église dans la chasse aux sorcières. Plus que jamais le corps sorcier s'avère nécessaire à la construction et au renforcement d'un autre grand corps collectif qui est celui de l'Église et sans doute aussi celui des États naissants. Le dominicain Jean Nider n'exprimait pas autrement la nécessité du corps sorcier par rapport à l'exaltation du corps légitime du Christ et de l'Église quand il affirmait dans un passage clé de son Formicarius:

Il est ainsi clair que la clémence et la sagesse de Dieu tout puissant qui s'étend avec force d'un bout du monde à l'autre, règlent même avec douceur les maléfices des démons et des pires des hommes, de telle sorte que dans le même temps où ils cherchent, par leur incrédulité, à affaiblir et à diminuer la foi et le royaume du Christ, ils les raffermissent et les enracinent plus fortement dans le cœur des hommes ${ }^{50}$.

Franck Mercier

Université de Rennes 2 - Crhisco-Cerhio.

\footnotetext{
${ }^{48} \mathrm{M}$. Van der Lugt, Le ver, le démon et la vierge. Les théories médiévales de la génération extraordinaire, Paris, Les Belles Lettres, 2004. Voir aussi «Les Anges et la magie au Moyen Âge » (actes de la table-ronde, Nanterre, 8-9 décembre 2000), Mélanges de l'École française de Rome, Moyen-Age, t. 114, 2, 2002.

${ }^{49}$ Dès le milieu du XII ${ }^{\mathrm{e}}$ siècle avec Jean de Salisbury, voir par exemple T. Struve, «The importance of the Organism in the Political Theory of John of Salisbury », dir. M. Wilks, The World of John of Salisbury, Studies in Church History, Subsidia, Oxford, 3, 1984, p. 303-317.

${ }^{50}$ Jean Nider, Formicarius, op. cit., p. 183.
} 